\title{
Challenges and Solutions on Interference Management in D2D System of Cellular Network
}

\author{
NITSR AT IAHAN ${ }^{1}$ ASHIKIIR RAHMAN KHAN ${ }^{1,}{ }^{*}$ MAIN IIDNIN $^{1}$ \\ MAHAMUDUL HASAN RANA ${ }^{2}$ \\ ${ }^{1}$ Department of Information and Communication Engineering \\ ${ }^{2}$ Department of Computer Science and Telecommunication Engineering \\ Noakhali Science and Technology University, Noakhali-3814 \\ BANGLADESH
}

\begin{abstract}
A single bidirectional link is used to allow communication between two devices in the device-todevice (D2D) communication system. D2D technology has to implement with the current cellular system. As both users D2D and cellular use the same licensed spectrum for transmission the chances of interferences increases. It is challenging for researchers to find out the proper mechanism to decrease interference and maximize performances. In this paper, we try to survey the challenges and their solutions to enable D2D communication in the cellular network with low interference. Here we describe the peer discover, mode selection process and interference management with power control and resource allocation. Finally, we can say that with proper power control, spectrum slicing and resource allocation we can mitigate co-tier and cross-tier interferences.
\end{abstract}

Key-Words: - Peer discover; Mode selection; Interference control; Resource allocation; Power control.

Received: March 13, 2020. Revised: November 16, 2020. Accepted: December 9, 2020. Published: December 26, 2020.

\section{Introduction}

D2D communication can occur if the devices are in

Device-to-Device (D2D) communication permits two near devices to speak with one another within the licensed cellular bandwidth while not a base station (BS) concerned or with restricted BS involvement. Typically, communicating D2D devices are located close to each other. Low-power and energy-efficient transmission are achievable because of this physical proximity. On the other hand, Spectrum efficiency can be improved because of two reasons.

The first one is that when two UEs are communicating in D2D mode, only one communication link is required instead of two, as shown in Figure 1.

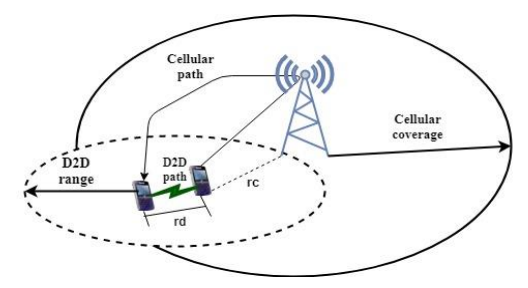

Fig.1. D2D communication under cellular network
D2D range and the D2D link distance (rd) is less than the cellular link distance (rc) otherwise cellular communication occur.

The second reason is called resource reuse that said that it is possible to allocate the same radio resources for cellular as well as D2D links in a cell. The fifth-generation cellular networks enable with D2D communication is taken into account as twotire networks [1]. The 2 tires during this networks square measure the macro-cell tire and also the device tire. Standard cellular communication is supported by the macro cell tire and D2D communication is supported by the device tire. The 2 tires' cellular network has an advantage over the standard cellular design like one-hop communication, optimization of power level, spectrum reusability, improve coverage space, etc. D2D communications can surface either overlaying or underlying in-band a cellular network or outband. Within the overlay case, D2D communications use dedicated resources, whereas within the underlay case D2D communications share common resources with the residual cellular network. 
During this analysis paper, we are going to specialize in the underlay policy, wherever the cellular and also the D2D users share common radio resources, organized into resource blocks (RBs). RBs are a coalescence of frequency and time symbols which are allocated to the users of the network [2]. In this analysis paper, we are going to study multicell interference management in D2D communication. In multi-cell surrounding multiple neighboring cells exist for serving the users of the network. Interference is lessened by power management, followed by the best resource allocation. Several researchers adopt different approaches for the mitigation of interference between D2D connections and cellular links.

Interference situation of a cellular system allowing D2D communications makes the role of power control and resource allocation in the system becomes more vital. It is important to notice that the level of interference caused by D2D links depends on the allocated resource and transmit power of each UE. Therefore, there is a great need of investigating how power control and resource allocation should be done in a cellular system when D2D links are present, as well as how they would affect the spectrum and energy efficiency of the system.

Our contributions are summarized in the following: (i) to show a peer discovery algorithm depends on the distance (ii) to present an optimization problem for mood selection in D2D based on TDMA model (iii) to analyze an effective minimum interference resource allocation algorithm.

\section{Literature Survey}

The fifth-generation (5G) cellular network is heading towards the device central approach, network setup, and managed by the devices themselves. D2D communication is being thought of as a vital element of the 5G networks. Many analyses have been dispensed within a previous couple of years to attain the total edges of D2D communication.

In 2014 apiece on D2D communication was done by the authors [3]. They illustrate that 2-tire network has to be designed with sensible interference management methods and applicable resource allocation schemes so there'll be a bottom impact on the performance of existing macro-cell BSs. This work offers an outline of two tire networks and describes different kinds of D2D communication supported the degree of the involvement of the cellular operator. Some rating schema for various varieties of device relaying was conjointly delineated during this work. However, they don't show how we've to allot resources and the way to manage interference once cellular users and D2D users are within the same spectrum.

Efficient assignment and power control of RBs are of fundamental importance in multi-cell interference management. Power control effectively mitigates interference with cellular networks. In 2017, a study is achieved by concentrating on power optimization [4]. With minimum SINR criteria and worst-case ICI cap in multiple neighboring cells they jointly show how to maximize the power. IEEE's members did another piece of work in 2015 [5].

The author takes into account a D2D underlying communication network for canceling interference, along with the transmission powers to maximize network utility. The users enjoy a significant gain regarding spectral efficiency. Resource management policy can be centralized or distributed in D2D communication. In traditional cellular networks, the eNB must effectively and timely determine the assignment of RBs to the network users. For $5 \mathrm{G}$ networks and to enable users D2D communication capabilities, the respective RB allocation approaches can be found in [6]. The authors propose scheduling algorithms for resource block (RB) allocation problems in D2D. This enabled networks to take into account the delay requirement of each user. Here, in compliance with the 3rd Generation Partnership Project (3GPP) standards, the eNB must assess the appropriate distribution of resources to users at the time scales of milliseconds, including devices that can communicate directly. The game players are the BS's and compete with each other to represent less dangerous D2D users.

Nevertheless, the game's presence of the Nash Equilibrium (NE) point is not analytically confirmed and is tested only through computational simulations. The prior research focuses on optimizing the total amount of prices in the network through game-theoretical approaches and resource pricing. Investigative work was published in 2016[7]. Both RB and power distribution problems are discussed here. The authors initially seek to reduce the interference caused by the D2D connections to the network's cellular connections, and then a power management strategy is applied to optimize the overall system throughput. Here we study some challenges and their solutions that have to face during D2D system implementation.

\section{Peer discover and mood selection}

If the link between the devices is favorable for D2D communication and they know each other identity then they can be considered as a D2D pair. Devices can discover themselves through message exchange. 
This message contains information about the transmitter, receiver and also used to estimate the path gain. There are some criteria SINR threshold, synchronization and network model that have to satisfy during message exchange. To discover the device if follow semi-centralized semi-networkdependent discovery algorithm rather than a Centralized fully network-dependent discovery algorithm then it takes less time concerning to message transmission [8]. The Semi-centralized semi-network-dependent discovery algorithm provides information regarding the path gains of the links between most entities in the cell. This information enables it to determine the mode.

Some performance objective like low latency, high spectral efficiency or low transmission power achievement depends on the selection of the perfect mode. The D2D mode has been chosen if the biased D2D link quality may be a minimum of just about pretty much as good as a result of the cellular transmission quality. The network or the UEs can be used to select mode. The UEs can operate in the cellular mode if the BS satiation serving quality is better than D2D. Generally, mode selection couple with power control because there are some sophisticated objectives like minimum QoS of the receiver, maximum transmit power, channel gain, optimal spectrum reuse, etc. are consider to take the decision. Using instantaneous system information or statistical system information the analysis would be done. This process leads to take optimal decisions at a given instance [9].

$$
\text { mode }=\left\{\begin{array}{cl}
\text { cellular, } & \text { if } P^{t}=\max \left(P^{r x}\right)>\beta \\
D 2 D, & \text { otherwise }
\end{array}\right.
$$

Here $P^{r x}$ is the receive signal strength from the base station and $\beta$ is the specific threshold value.

\subsection{Interference management scheme}

Co-tier and Cross-tier interferences are occurs in a 2-tier or 3-tier cellular system. In 3-tier cellular network possible interferences are designed in Figure 2.

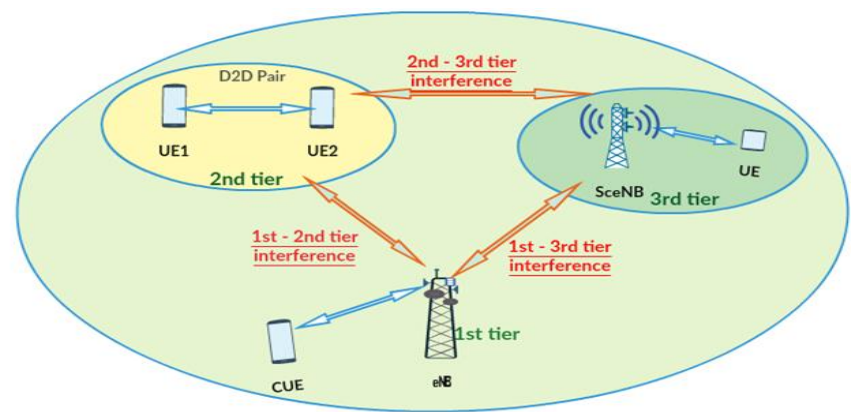

Fig. 2 Interference in D2D communication in 3tier network architecture

Here we see 1st-tier and 2nd-tier interference as users in the 2nd cell available for both mode D2D and cellular, a similar situation also can create interference between 2nd-tier and 3rd tier and between 3 rd-tier and 1st-tier. To reduce those interferences any of the management systems among centralized, distributed and semi-distributed can be used. Stochastic theory, game theory, graph theory, evolution theory, optimization theory, queuing theory, etc. mathematical computations are performed for analysis and design of interference management in D2D schema. Several research works have been done based on resource reuse, power control and spectral splitting to coordinate interference. Cancellation of interference, avoidance of interference and coordination of interference are three interference management scheme. The short description of the interference management scheme in D2D communications is shown in Table 3.

Table 1 Brief summary of interference management scheme in D2D communication.

\begin{tabular}{|c|c|c|c|}
\hline $\begin{array}{l}\text { Ref. } \\
\text { No. }\end{array}$ & $\begin{array}{c}\text { Algorithm or } \\
\text { procedure } \\
\text { Used }\end{array}$ & Found & $\begin{array}{c}\text { Interference } \\
\text { management } \\
\text { scheme }\end{array}$ \\
\hline [10] & $\begin{array}{l}\text { Implements a } \\
\text { bucket-based } \\
\text { degree of } \\
\text { freedom (DoF) } \\
\text { algorithm for } \\
\text { an efficient } \\
\text { configuration } \\
\text { of antenna. }\end{array}$ & $\begin{array}{l}\text { The } \\
\text { throughput } \\
\text { was } \\
\text { increased } \\
\text { using } \\
\text { multiuser } \\
\text { D2D } \\
\text { System }\end{array}$ & $\begin{array}{l}\text { Avoidance } \\
\text { scheme }\end{array}$ \\
\hline
\end{tabular}

(MD2D) 


\begin{tabular}{|c|c|c|c|c|c|c|c|}
\hline $\begin{array}{l}\text { Ref. } \\
\text { No. }\end{array}$ & $\begin{array}{l}\text { Algorithm or } \\
\text { procedure } \\
\text { Used }\end{array}$ & Found & $\begin{array}{l}\text { Interference } \\
\text { management } \\
\text { scheme }\end{array}$ & $\begin{array}{l}\text { Ref. } \\
\text { No. }\end{array}$ & $\begin{array}{l}\text { Algorithm or } \\
\text { procedure } \\
\text { Used }\end{array}$ & Found & $\begin{array}{c}\text { Interference } \\
\text { management } \\
\text { scheme }\end{array}$ \\
\hline [11] & $\begin{array}{l}\text { Proposed fully } \\
\text { distributed } \\
\text { random access } \\
\text { protocol that } \\
\text { prevent the } \\
\text { interferers from } \\
\text { transmitting a } \\
\text { signal around } \\
\text { the D2D } \\
\text { receiver. }\end{array}$ & $\begin{array}{l}\text { The proposed } \\
\text { distributed } \\
\mathrm{D} 2 \mathrm{D} \text { scheme } \\
\text { is performing } \\
\text { better than } \\
\text { the } \\
\text { traditional } \\
\text { distributed } \\
\mathrm{D} 2 \mathrm{D} \text { scheme. }\end{array}$ & $\begin{array}{l}\text { Avoidance } \\
\text { scheme }\end{array}$ & [16] & $\begin{array}{l}\text { Proposed } \\
\text { a graph- } \\
\text { coloring-based } \\
\text { resource } \\
\text { sharing } \\
\text { (GCRS) } \\
\text { scheme }\end{array}$ & $\begin{array}{l}\text { for cellular } \\
\text { communicati } \\
\text { on. } \\
\text { optimize the } \\
\text { spectrum } \\
\text { utilization }\end{array}$ & $\begin{array}{l}\text { Interference } \\
\text { coordination } \\
\text { schemes }\end{array}$ \\
\hline [12] & $\begin{array}{l}\text { To maximize } \\
\text { the } \\
\text { transmission } \\
\text { capacity with } \\
\text { guaranteed link } \\
\text { QoSs proposed } \\
\text { a feasible set } \\
\text { scheme. }\end{array}$ & $\begin{array}{l}\text { Reduce the } \\
\text { average risk } \\
\text { of an outage } \\
\text { by up to } 92 \%\end{array}$ & $\begin{array}{l}\text { Interference } \\
\text { coordination } \\
\text { schemes }\end{array}$ & [17] & $\begin{array}{l}\text { Proposed } \\
\text { power control } \\
\text { and resource } \\
\text { allocation } \\
\text { algorithm } \\
\text { based on the } \\
\text { interference }\end{array}$ & $\begin{array}{l}\text { In terms of } \\
\text { the sum data } \\
\text { rate and } \\
\text { coverage } \\
\text { probability, } \\
\text { outperform } \\
\text { the }\end{array}$ & $\begin{array}{l}\text { Interference } \\
\text { coordination } \\
\text { schemes }\end{array}$ \\
\hline [13] & $\begin{array}{l}\text { Multiple } \\
\text { interference } \\
\text { cancellation } \\
\text { (MIC) } \\
\text { techniques for } \\
\text { nonorthogonal } \\
\text { multiple access } \\
\text { (NOMA) }\end{array}$ & $\begin{array}{l}\text { Considering } \\
\text { better } \\
\text { performance } \\
\text { and low } \\
\text { complexity } \\
\text { MIC } \\
\text { is better than } \\
\text { successive } \\
\text { interference } \\
\text { cancellation } \\
\text { (SIC) }\end{array}$ & $\begin{array}{l}\text { Interference } \\
\text { cancellation }\end{array}$ & [18] & $\begin{array}{l}\text { limited area } \\
\text { (ILA). } \\
\text { Based on } \\
\text { Guard zone by } \\
\text { enabling the } \\
\text { capacity of the } \\
\text { BS's to } \\
\text { successively } \\
\text { cancel } \\
\text { interference. }\end{array}$ & $\begin{array}{l}\text { conventional } \\
\text { scheme } \\
\text { The average } \\
\text { throughput of } \\
\text { a user } \\
\text { equipment } \\
\text { and the } \\
\text { successful } \\
\text { transmission } \\
\text { probability } \\
\text { improved }\end{array}$ & $\begin{array}{l}\text { Interference } \\
\text { cancellation }\end{array}$ \\
\hline [14] & $\begin{array}{l}\text { By modifying } \\
\text { the } \\
\text { constellation } \\
\text { rotation } \\
\text { technique, a } \\
\text { security- } \\
\text { embedded } \\
\text { interference } \\
\text { avoidance }\end{array}$ & $\begin{array}{l}\text { The privacy } \\
\text { aspect of the } \\
\text { security issue } \\
\text { between } \\
\text { cellular users } \\
\text { and D2D } \\
\text { users was } \\
\text { highlighted. }\end{array}$ & $\begin{array}{l}\text { Avoidance } \\
\text { techniques }\end{array}$ & [19] & $\begin{array}{l}\text { Based on a } \\
\text { harvesting data } \\
\text { approach in a } \\
\text { multihop D2D } \\
\text { communication } \\
\text { s community } \\
\text { proposed a P2P } \\
\text { system called } \\
\text { "Lunius" }\end{array}$ & $\begin{array}{l}\text { optimizes } \\
\text { P2P local- } \\
\text { file-sharing } \\
\text { and assure } \\
\text { QoS } \\
\text { requirements } \\
\text { of user } \\
\text { equipment }\end{array}$ & $\begin{array}{l}\text { Interference } \\
\text { cancellation }\end{array}$ \\
\hline
\end{tabular}

[15] A radio resource established allocation D2D links scheme was can reuse the proposed for cellular band multihop D2D and still connections in produce Avoidance LTE-A minimum techniques networks. interference

\subsection{Power control}

The proper power control mechanism can suppress co-channel interference between D2D users and cross-channel interference for both uplink and downlink case. The author in [20] proposed a distributed on-off power control algorithm for both centralized and decentralized systems. In this schema, the D2D transmitter select transmit 
power from the decision set $\left\{0, P_{\max , d}\right\}$ based on their channel gain, the direct link information and nonnegative threshold $G_{\text {min }}$. $G_{\min }$ is known by all users and plays an important role to determine the sum-rate performance. Large $G_{\min }$ reduce interference but scheduled smaller D2D users. So it was a good choice to optimize the $G_{\min }$ with transmission probability $\left(P_{S}\right)$ and maximize the sum rate performance. Using stochastic geometry and assuming that the uplink user transmission power is independent they analyzed the coverage probability. Optimal cellular link power control strategy follow some conditions: i) the cellular users (CUE) used the constant transmit power if the user is located around the cell center ii) the CUE follow the on-off power control strategy and increases its transmit power if the user is located at the midrange of the cell edge iii) the CUE sends a signal with its maximum transmit power if the user is located at around the cell edge. In this condition, the probability of coverage decreased linearly. With the characterized ergodic sum-rate of the D2D link, they optimize the D2D on off threshold and maximize the approximated transmission capacity of the D2D link.

Coverage probability was compared with the sparse D2D link and dense D2D link. The cellular coverage probability increase with low target SINR values in the sparse scenario.

Because of the increased intra-D2D link, interference D2D user coverage probability decreased in the dense scenario. But still, the total number of successful D2D transmission was larger than the sparse D2D link scenario.

Binary power control scheme (BPCS) with complexity $\mathrm{O}\left[\mathrm{K}\left\{\mathrm{N}+\left(\mathrm{K}^{*} \mathrm{M}\right)\right\}\right]$ was proposed by the author in [21]. This proposed algorithm can achieve a near-optimal solution for a multicell communication environment when multiple D2D pairs simultaneously reuse the same cellular resource. Only if the channel quality is sufficient then the transmission is initiated in BPCS. The transmit power of the users can be increased until it satisfies the required SINR. Upon reaching the maximum usable power and attaining the appropriate SINR value the process will end.

\subsection{Resource allocation}

Here the term "resources" depends on the channel access scheme. For instance, "resources" would mean time-slots in time division multiple access (TDMA) systems or frequency bands in frequency division multiple access (FDMA) systems. The concept of radio resources in TDMA and FDMA systems is best explained in Figure 3.

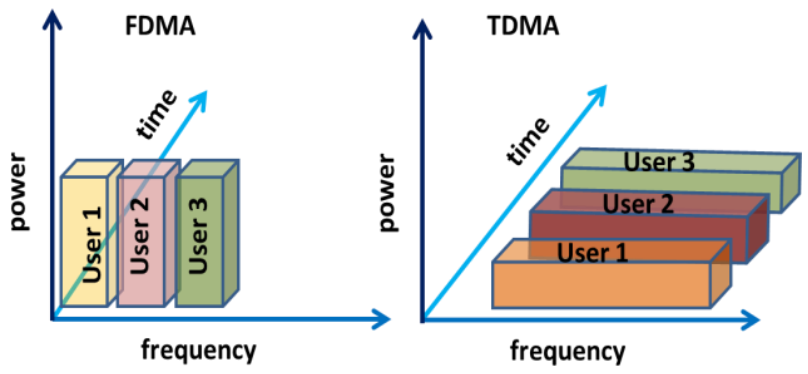

Fig.3. FDMA and TDMA radio resource

In FDMA frequency band has been divided into disjoint sub-band and in TDMA the time has been divided into non overlapping time slot.

Radio resource allocation means figuring out how radio resources should be allocated to meet the instantaneous capacity and QoS demand of the users.

For each communication link (either cellular or D2D) sufficient resource blocks must be allocated. The number of required resource blocks can vary according to the application.

Video streaming applications, for instance, would require more resources than email applications.

If possible, orthogonal resources should be allocated to all links so that no intra-cell interference will be generated. However, this cannot happen if there are not enough orthogonal resources. In this situation, resource reuse is necessary. This is a problem because UEs sharing the same radio resource generate interference to each other. Due to the random positions of the UEs, the selection of resources for each link becomes critical as it will affect the interference generated to other links sharing the same resource. This is where resource allocation algorithms come into play.

The authors in [22] proposed and analyzed a minimum interference algorithm that assumes that all path gains for cellular and D2D links are known. Using the path gains, interference generated to and received from other links sharing the resource can be estimated. Interference generated to other links is estimated as the sum of path gains between the D2D transmitter to all receivers sharing the same resource. Interference received from other links is 
estimated as the sum of path gains between the D2D receiver to all transmitters sharing the same resource.

Let $G_{t, r, j}$ be the path gains between transmitter- $t$ and receiver-r using resource- $\mathrm{j}$. For a given resource $\mathrm{j}$, the following quantities are defined for each D2D link $l$.

$$
\begin{aligned}
& G_{\text {InterferenceGenerated }, l, j}=\sum_{r} G_{l, r, j} \\
& G_{\text {InterferenceReceived }, l, j}=\sum_{t} G_{t, r, j}
\end{aligned}
$$

To clarify what GInterferenceGenerated and GInterferenceReceived mean, consider a cell having two resource blocks called RB1 and RB2. Initially, the cell has one cellular UE transmitting using RB1 and one D2D pair transmitting using RB2. A D2D candidate is detected in the system. The resource allocation algorithm must decide whether this D2D candidate should reuse RB1 or RB2.In Figure 4 path gains in the system is illustrated.

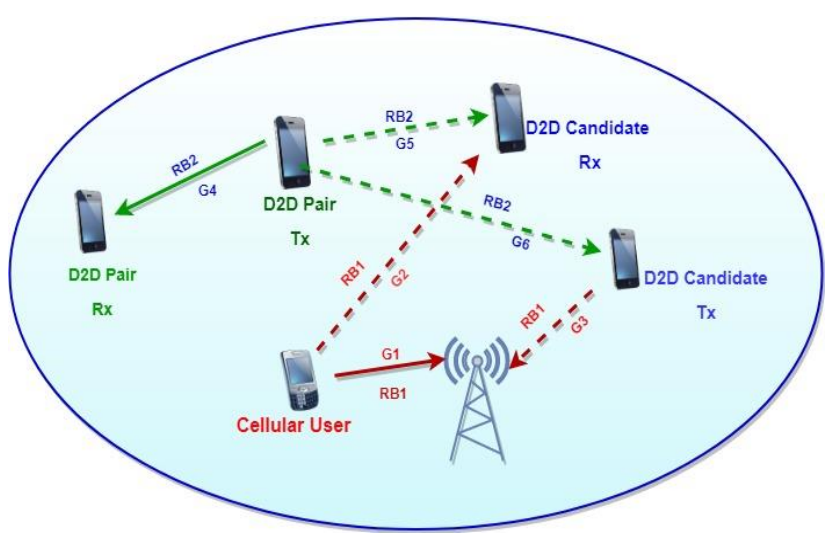

$\longrightarrow$ D2D link,---- Interference link

Tx: Transmitter, Rx: Receiver, Gi: Path gain

Fig.4 Path gains considered in MinInterfalgorithm

If the D2D candidate uses RB1, its transmitter will generate interference to the base station, and its receiver will receive interference from the cellular UE.

Hence, $\quad G_{\text {InterferenceGenerated,RB1 }}=G_{3}$ and $G_{\text {InterferenceReceived, } R B 1}=G_{2}$.

If the D2D candidate uses RB2, its transmitter will generate interference to the existing D2D pair's receiver, and its receiver will receive interference from the existing D2D pair's transmitter.

Hence,

$$
\text { and } \quad \begin{aligned}
& G_{\text {InterferenceGenerated }, R B 2}=G_{6} \\
& G_{\text {InterferenceReceived }, R B 2}=G_{5} .
\end{aligned}
$$

To avoid those interference minimum interference algorithm allocates orthogonal resources for all cellular users randomly and if any orthogonal resource left then they will allocate for each D2D user. If there are no remaining resources left then any available resource $\mathrm{j}$ with minimum value $\mathrm{S}(\mathrm{j})=$ is used by the D2D [GInterferenceGenerated, $j$ + GInterferenceReceived, $j$ ] candidate to transmit in D2D mode

Greedy-based mode selection and channel allocation algorithm was proposed in [23]. With number of cellular links (M) and number of D2D links (N) computational complexity of the mode selection and channel allocation step is $\mathrm{O}(2 \mathrm{MN})$. According to the sum transmission data rates allocate appropriate channel resource for D2D links, but also switch the communication mode for D2D links flexibly.

In terms of sum-rate, the joint mode selection (MS), resource allocation (RA), and scheduling algorithm can finds minimal SINR knowledge and significant performance improvement. The author [24] proposed a greedy heuristic algorithm that properly assigns the available resources among cellular users and D2D pairs. The proposed system improves sum rate and average UE SINR, with no increase in signalling overhead or complexity.

Using successive interference cancellation (SIC) decoding to eliminate interference between cellular users and D2D pairs the authors in [25] proposed an interlay mode for the nonorthogonal multiple access (NOMA) system which allows power domain multiplexing between them. Proposed a graphbased algorithm by applying branch-and-bound method to obtain optimal solution to tackle the combinatorial nature of mode selection and spectrum assignment. To increase the system sum rate and at the same time fulfil the SIC decoding constraint they proposed a resource allocation scheme and a joint D2D mode 
selection that makes up as a combinatorial optimization problem.

\section{Conclusion}

With performing this analysis the main question we want to find is: How to create a suitable D2D communication scheme with low interference? The popularity of mobile communications has increased immensely in the past decade. We argued that mobile operators need innovative solutions to cope with the rising demand for data traffic and that leads us towards the $5 \mathrm{G}$. Our main focus study topic was D2D communications in an emergency scenario. We explained the concept of the device to device communications briefly by knowing the major steps to originate a D2D call like peer discovery and the methods used for peer discovery, mode selection, resource allocation, interference management, power control and necessary spectrum for D2D communication in the cellular network. We show the peer discover technique, mode selection algorithm and the resource allocation and power control technique to mitigate interference. The algorithms are based on the emergency scenario to select D2D pair. Based on the TDMA mode we saw a minimum interference resource allocation algorithm. We also discuss the result of the proposed systems of the authors. Finally, we saw a realistic urban cell scenario for peer discovery and selection of D2D pair in abnormal conditions without the usage of the Base station. We realize that we can think more about introducing a new era that mobile devices can do the work of the networks on their own.

\section{References:}

[1] Asadi, A. Wang, Q. Mancuso, V. "A survey on device-to-device communication in cellular networks.", IEEE Commun. Surv. Tutor. , 2014.

[2] Datsika, E.; Antonopoulos, A. Zorba, N. Verikoukis." Cross-Network Performance Analysis of Network Coding Aided Cooperative Outband D2D Communications". IEEE Trans. Wirel. Commun. 2017.

[3] Mohsen Nader Tehrani, Murat Uysal, and Halim Yanikomeroglu. "Device-to-Device

Communication in 5G Cellular Networks: Challenges, Solutions, and Future Directions.",

IEEE Communications Magazine, 2014.

[4] Ali Ramezani-Kebrya, Min Dong, Ben Liang, Gary Boudreau and S. Hossein Seyedmehdi. "Joint Power Optimization for Device-to-Device Communication in Cellular Networks with
Interference Control.", IEEE Transactions on Wireless Communications. ,2017.

[5] Zhou, Liang, Kalle Ruttik, and Olav Tirkkonen. "Interference Canceling Power Optimization for Device to Device Communication.", IEEE, 2015.

[6] Lioumpas, A.S. Alexiou, "Uplink scheduling for Machine-to-Machine communications in LTE-based cellular systems."IEEE GLOBECOM Workshops (GC Wkshps), Houston, TX, USA, 2011.

[7]Jiang, F.Wang, B.C.; Sun, C.Y.; Liu, Y.; Wang, $\mathrm{X}$., "Resource allocation and dynamic power control for D2D communication underlaying uplink multicell networks", Wireless Network, 2016.

[8] Anastasios Thanos, Serveh Shalmashi, and Guowang Miao, 'Network-assisted discovery for device-to-device communications", IEEE Globecom Workshops (GC Wkshps), 2013.

[9] H. ElSawy, E. Hossain, M.-S. Alouini, "Analytical modeling of mode selection and power control for underlay $\mathrm{d} 2 \mathrm{~d}$ communication in cellular networks", IEEE Trans. Commun., 2013.

[10] Chiu, S.L. Lin, K.C.-J., Lin, G.-X., Wei, H.-Y. "Empowering Device-to-Device networks with cross-link interference management." IEEE Trans. Mob. Comput. 2017.

[11] Zihan, E., Choi, K.W., Kim, D.I. "Distributed random access scheme for collision avoidance in cellular Device-to-Device communication." IEEE Trans. Wirel. Commun. 2015.

[12] Xu,Y., Liu,F., Wu,P. "Interference management for $\mathrm{D} 2 \mathrm{D}$ communications in heterogeneous cellular networks." Pervasive Mob. Comput. 2018.

[13] Gandotra P., J ha,R.K.,Jain,S. "Green NOMA with multiple interference cancellation(MIC) using sector-based resource allocation." IEEE Trans. Netw. Serv. Manag. 2018.

[14] Sun L., Du Q., Ren, P., Wang Y. "Two birds with one stone: Towards secure and interferencefree D2D transmissions via constellation rotation." IEEE Trans. Veh. Technol. 2015.

[15] Melki L., Najeh,S., Besbes,H. "Radio resource management scheme and outage analysis for network-assisted multi-hop D2D communications." Digit. Commun. Netw. 2016.

[16] Yang T., Zhang R.; Cheng X., Yang L. "Graph coloring based resource sharing (GCRS) scheme for D2D communications underlaying full-duplex cellular networks." IEEETrans. Veh. Technol. 2017. [17] Sun J., Zhang Z., Xiao H., Xing C. "Uplink interference coordination management with power control for D2D underlaying cellular networks: Modeling, algorithms, and analysis." IEEE Trans. Veh. Technol. 2018. 
[18] Lv S., Xing C., Long K., Zhang Z. "Guard zone based interference management for $\mathrm{D} 2 \mathrm{D}$-aided underlaying cellular networks." IEEE Trans. Veh. Technol. 2016.

[19] Wu, Y., Wang, S., Liu, W., Guo, W., Chu, X. "Iunius: A cross-layer peer-to-peer system with Device-to-Device communications." IEEE Trans. Wirel. Commun. 2016

[20] Namyoon Lee, Xingqin Lin, Jeffrey G. Andrews, and Robert W. Heath Jr. "Power Control for D2D Underlaid Cellular Networks: Modeling, Algorithms and Analysis", IEEE

Journal on Selected Areas in Communications, 2013.

[21] Devarani Devi Ningombam and Seokjoo Shin, "Optimal Resource Management and Binary Power Control in Network-Assisted D2D Communications for Higher Frequency Reuse Factor", Sensors (Basel), 2019.

[22] Marco Belleschi ,Gábor Fodor, Demia Della Penda, Aidilla Pradini, Mikael Johansson, Andrea Abrardo, "Benchmarking Practical RRM Algorithms for D2D Communications in LTE Advanced", Wireless Personal Communications, 2014.
[23] Tian, C., Qian, Z., Wang, X., Hu, L. “ Analysis of joint relay selection and resource allocation scheme for relay-aided D2D communication networks." IEEE Access 2019.

[24] Bithas P.S., Maliatsos K., Foukalas F. "An SINR-aware joint mode selection, scheduling, and resource allocation scheme for D2D

communications." IEEE Trans. Veh. Technol. 2019. [25] Dai Y., Sheng M., Liu, J., Cheng N., Shen, X., Yang Q. "Joint mode selection and resource allocation for D2D-enabled NOMA cellular networks." IEEE Trans. Veh. Technol. 2019

\section{Author Contributions:}

Nusrat Jahan has carried out the work, analysis and simulation. Jahan also has prepared the manuscript.

Md. Ashikur Rahman Khan has contributed to monitoring the entire work and reviewed the manuscript.

Main Uddin has performed the review and editing work.

A.R. Mahmududl Hasan has performed the formulation of the research goal.

\section{Creative Commons Attribution License 4.0 (Attribution 4.0 International, CC BY 4.0)}

This article is published under the terms of the Creative Commons Attribution License 4.0

https://creativecommons.org/licenses/by/4.0/deed.en US 